\title{
NORM DECREASING HOMOMORPHISMS OF MEASURE ALGEBRAS
}

\author{
BY \\ ROGER RIGELHOF
}

1. Introduction. Let $G$ be a locally compact group (=locally compact Hausdorff topological group). By the measure algebra of $G$ we mean the Banach *-algebra $M(G)$ of bounded regular Borel measures on $G$. It is well known that $M(G)$ is the dual of $C_{0}(G)$ the Banach space of continuous complex-valued functions on $G$ which "vanish at infinity". The group algebra $L^{1}(G)$ is the $*$-subalgebra of $M(G)$ consisting of all measures that are absolutely continuous with respect to the Haar measure on $G$. Alternatively $L^{1}(G)$ can be defined as the Banach *-algebra of (equivalence classes of) Haar summable functions on $G$.

Let $F$ and $G$ be locally compact groups, $\alpha$ a bicontinuous isomorphism of $F$ onto $G$, and $\gamma$ a character on $F$. For $\mu$ in $M(F)$ and $f$ in $C_{0}(G)$ let $T \mu(f)=\mu(\gamma(f \circ \alpha))$. Then the mapping $\mu \mapsto T \mu$ is an isometric $*$-isomorphism of $M(F)$ onto $M(G)$ (Lemma 2). In $\S 3$ we show that every norm decreasing isomorphism of $M(F)$ onto $M(G)$ is of the above form and consequently is an isometric *-isomorphism (Theorem 1). A number of other results follow from this, in particular Wendel's theorem on norm decreasing isomorphisms of group algebras.

Theorem 1 generalizes a result of B. E. Johnson [7] on isometric isomorphisms of measure algebras. In [7] Johnson used norm properties of measures in $L^{1}(F)$ to show that each isometric isomorphism maps $L^{1}(F)$ onto $L^{1}(G)$ and consequently by applying Wendel's result it follows that each isometric isomorphism is of the form described above. Our generalization of Johnson's result has the advantage that Wendel's theorem is a consequence.

In $\S 4$ we use the results of $\S 3$ to prove a similar structure theorem for norm decreasing homomorphisms $T$ of $M(F)$ onto $M(G)$ such that $T\left(\mu * L^{1}(F)\right)=\{0\}$ implies $T \mu=0$. (In this case the bicontinuous isomorphism of $F$ onto $G$ mentioned above becomes a continuous and open homomorphism of $F$ onto $G$.)

The final section of the paper is concerned with bipositive homomorphisms of $M(F)$ onto $M(G)$. Here we show that the hypothesis of norm decreasing may be dropped provided that the homomorphism maps the positive cone onto the positive cone.

The first three sections of this paper formed a part of the author's $\mathrm{Ph}$. D. thesis at McMaster University. The author acknowledges with thanks the supervision given him by Professor Taqdir Husain.

Presented to the Society, May 15, 1967 under the title Isomorphisms of measure algebras; received by the editors November 13, 1967. 
2. Preliminaries. Let $(X, \tau)$ be a topological space, we write $X_{\tau}$ in place of $(X, \tau)$ and if $Y \subseteq X, Y_{\tau}$ is $Y$ with the relative $\tau$-topology. If $\left(x_{j}: j \in J\right)$ is a net in $X$ which converges to $x$ we shall write this as $x_{j} \rightarrow x$. The $\tau$-closure of a subset $Y \subseteq X$ is written $\mathrm{Cl}_{\tau} Y$.

Let $G$ be a locally compact group, $C(G)$ is the Banach space of bounded continuous complex valued functions on $G, K(G)$ the subspace of $C(G)$ consisting of functions whose support is compact. $C_{0}(G)$ may be defined as the closure of $K(G)$ in $C(G)$.

As remarked in the introduction, $M(G)$ is the dual of $C_{0}(G)$. The weak topology on $M(G)$ is the $\sigma\left(M(G), C_{0}(G)\right)$-topology, i.e. the coarsest topology on $M(G)$ such that for each $f$ in $C_{0}(G)$ the mapping $\mu \mapsto \mu(f)$ of $M(G)$ into the complex field is continuous. We shall frequently write $\sigma$ in place of $\sigma\left(M(G), C_{0}(G)\right)$.

For $x$ in $G, \varepsilon_{x}$ is the Dirac measure at $x$, i.e. the element of $M(G)$ defined by $\varepsilon_{x}(f)=f(x)$ for $f \in C_{0}(G) . G^{\varepsilon}$ is the collection of all Dirac measures.

If $\mu$ is any measure on $G$, then we shall use $\mu$ for the corresponding integral, writing $\mu(f)=\int f d \mu$ for any $\mu$-integrable function $f$.

The support of a measure $\mu$, written $\operatorname{Supp}(\mu)$ is the set of all $x$ in $G$ such that for each neighborhood $U$ of $x$, there is an $f$ in $K(G)$ which vanishes outside $U$ with $\mu(f) \neq 0$.

Proposition 1. (i) $G$ is isomorphic and homeomorphic to $G_{\sigma}^{\varepsilon}$.

(ii) The mappings $\mu \mapsto \mu * \lambda, \mu \mapsto \lambda * \mu$ and $\mu \mapsto \mu^{*}$ of $M(G)$ into itself are $\sigma\left(M(G), C_{0}(G)\right)$-continuous.

(iii) Let $V$ be the linear span of $G^{\varepsilon}$. Then for any $\mu \in M(G)$,

$$
\mu \in \mathrm{Cl}_{\sigma}\{\lambda \in V:\|\lambda\| \leqq\|\mu\| \text { and } \operatorname{Supp}(\lambda) \subseteq \operatorname{Supp}(\mu)\} .
$$

Proof. (i) It is straightforward to see that the mapping $x \mapsto \varepsilon_{x}$ of $G$ onto $G^{\varepsilon}$ is a bicontinuous isomorphism.

(ii) For $f \in C_{0}(G)$, define $\lambda^{-}(f)$ by $\lambda^{-}(f)(x)=\lambda\left({ }_{x} f\right)$ where ${ }_{x} f(y)=f(x y)$. Then $\lambda^{-}(f) \in C_{0}(G)$ [6, p. 264], and $\mu * \lambda(f)=\mu\left(\lambda^{-}(f)\right)$. It follows that $\mu \mapsto \mu * \lambda$ is $\sigma\left(M(G), C_{0}(G)\right)$-continuous. Similarly the definition of $\mu^{*}$ yields the continuity of the mapping $\mu \mapsto \mu^{*}[6$, p. 299]. To show that $\mu \mapsto \lambda * \mu$ is continuous observe that this mapping can be written $\mu \mapsto \mu^{*} \mapsto \mu^{*} * \lambda^{*}=(\lambda * \mu)^{*} \mapsto(\lambda * \mu)^{* *}=\lambda * \mu$.

The proof of (iii) is the same as the proof of Corollary 1 on p. 71 of [2].

Corollary. Given any $\mu \in M(G)$ there is a net $\left(\mu_{j}: j \in J\right)$ in

$$
A=\{\lambda \in V: \operatorname{Supp}(\lambda) \subseteq \operatorname{Supp}(\mu) ;\|\lambda\| \leqq\|\mu\|\}
$$

such that $\mu_{j} \stackrel{\sigma}{\rightarrow} \mu$ and $\|\mu\|=\lim \left\|\mu_{j}\right\|$.

Proof. $\mu_{j} \stackrel{\sigma}{\rightarrow} \mu$ implies lim inf $\left\|\mu_{j}\right\| \geqq\|\mu\|$ since the mapping $\mu \mapsto\|\mu\|$ is lower semicontinuous in $M(G)_{\sigma}$.

Let $L^{1}(G)$ be the subset of $M(G)$ consisting of measures that are absolutely continuous with respect to the Haar measure on $G$. It is well known that $L^{1}(G)$ is a 
two-sided norm closed ideal in $M(G)$. Let $B\left(L^{1}(G)\right)$ be the algebra of all bounded linear operators which map $L^{1}(G)$ into itself, taken with the topology of simple convergence (i.e. the coarsest topology such that for each $\lambda \in L^{1}(G)$, the mapping $T \mapsto T \lambda$ is continuous. $L^{1}(G)$ is taken with the norm topology). For $\mu \in M(G)$ we define $T_{\mu} \in B\left(L^{1}(G)\right)$ by $T_{\mu} \lambda=\mu * \lambda$. The mapping $\mu \mapsto T_{\mu}$ is one-one. The sotopology on $M(G)$ is the coarsest topology such that this map is continuous. A theorem of Wendel [8] states that the image of $M(G)$ under this mapping is closed in $B\left(L^{1}(G)\right)$.

Proposition 2. (i) On norm bounded sets so $\supset \sigma\left(M(G), C_{0}(G)\right)$.

(ii) $G_{\sigma}^{\varepsilon}=G_{s o}^{\varepsilon}$.

(iii) The mappings $\mu \mapsto \mu * \lambda, \mu \mapsto \lambda * \mu$ are so-continuous.

(iv) Let $V$ be the linear span of the Dirac measures. For any $\mu \in M(G)$ we have $\mu \in \mathrm{Cl}_{\text {so }}\{\lambda \in V: \operatorname{Supp}(\lambda) \subseteq \operatorname{Supp}(\mu)$ and $\|\lambda\| \leqq\|\mu\|\}$.

(v) Let $\mu \in M(G)$ then $\mu \in \mathrm{Cl}_{\text {so }}\left\{\lambda \in L^{1}(G):\|\lambda\| \leqq\|\mu\|\right\}$.

Proof. (i) follows from Lemma 1.1.1 of [4] and the fact that any $f \in C_{0}(G)$ is left (and right) uniformly continuous [6, p. 185]. (ii) follows from (i) together with Proposition 1(i) and the fact that the mapping $x \mapsto \varepsilon_{x} * \lambda$ is continuous for $\lambda$ in $L^{1}(G)$. The proof of (iii) is an easy calculation. To prove (iv) use Lemmata 1.1.2 and 1.1.3 of [4]. The proof of (v) is an easy consequence of the facts that $L^{1}(G)$ is an ideal in $M(G)$ and $L^{1}(G)$ contains an approximate unit of norm 1 .

COROLlaRY. The mapping $\lambda \mapsto\|\lambda\|$ is lower semicontinuous in the so-topology.

The next proposition is the well-known relationship between the Dirac measures on $G$ and the extreme points of the unit ball in $M(G)$.

Proposition 3. The extreme points of the unit ball of $M(G)$ are precisely the measures $\gamma \varepsilon_{x},|\gamma|=1, x \in G$.

Proposition 4. Let $T$ be a norm bounded homomorphism of $M(F)$ onto $M(G)$ and suppose that for $\mu$ in $M(F), T\left(\mu * L^{1}(F)\right)=\{0\}$ implies $T \mu=0$. Then $T$ restricted to norm bounded sets is continuous as a mapping of $M(F)_{\text {so }}$ onto $M(G)_{\sigma}$.

Proof. Let $\left(e_{j}: j \in J\right)$ be an approximate unit in $L^{1}(F)$. By Lemma 4.1.2 of Greenleaf [4], $T e_{j} \stackrel{\sigma}{\rightarrow} \varepsilon$ where $\varepsilon$ is an idempotent such that $\varepsilon * \mu=\mu * \varepsilon=\mu$ for all $\mu$ in $\mathrm{Cl}_{\sigma} T\left(L^{1}(F)\right)$. Since $T$ is onto and $T\left(\mu * L^{1}(F)\right)=\{0\}$ implies $T \mu=0$, it follows that $\varepsilon$ is the unit of $M(G)$. Let $\left(\mu_{j}: j \in J\right)$ be a norm bounded net in $M(F)$ and suppose that $\mu_{j} \stackrel{\text { so }}{\rightarrow} \mu \in M(F)$. For any $\lambda$ in $L^{1}(F)$ we have $\mu_{j} * \lambda \rightarrow \mu * \lambda$ so that $T \mu_{j} * T \lambda \rightarrow T \mu * T \lambda$. Since $\left(T \mu_{j}: j \in J\right)$ is norm bounded and the unit ball of $M(G)$ is $\sigma\left(M(G), C_{0}(G)\right)$-compact it follows that there is a $\mu^{\prime} \in M(G)$ and a subnet $\left(T \mu_{j(i)}\right)$ such that $T \mu_{j(i)} \stackrel{\sigma}{\rightarrow} \mu^{\prime}$. Consequently for any $\lambda$ in $L^{1}(F)$ we have $T \mu * T \lambda$ $=\mu^{\prime} * T \lambda$, since multiplication is separately continuous in $M(G)_{\sigma}$. Thus $T \mu * T e_{j}$ $=\mu^{\prime} * T e_{j}$, and since $T e_{j} \stackrel{\sigma}{\rightarrow} \varepsilon$, we have $T \mu=\varepsilon * T \mu=\mu^{\prime}$. This completes the proof. 
LeMma 1. Let $F, G$ be locally compact groups and let $T$ be a norm decreasing homomorphism of $M(F)$ onto $M(G)$. Then for each $x \in F$ there is $a y \in G$, and $a$ complex number $\gamma,|\gamma|=1$ such that $T \varepsilon_{x}=\gamma \varepsilon_{y}$.

Proof. Let $L_{x}$ be the operator on $M(G)$ defined by $L_{x} \mu=T \varepsilon_{x} * \mu$. Since $T$ is a norm decreasing homomorphism of $M(F)$ onto $M(G)$ it follows that $L_{x}^{-1}$ exists; $L_{x}^{-1}=L_{x^{-1}}$ and $\left\|L_{x}\right\| \leqq 1,\left\|L_{x}^{-1}\right\|_{1} \leqq 1$. Let $\mu, \lambda \in M(G),\|\mu\| \leqq 1,\|\lambda\| \leqq 1$, and suppose that $T \varepsilon_{x}=a \mu+(1-a) \lambda$ for $0<a<1$. Then $\varepsilon_{e}=a L_{x}^{-1} \mu+(1-a) L_{x}^{-1} \lambda$, and since $\varepsilon_{e}$ is an extreme point of the unit ball, we have $\mu=\lambda$. Thus $T \varepsilon_{x}$ is an extreme point of the unit ball of $M(G)$ so now the lemma follows from Proposition 3.

\section{Norm decreasing isomorphisms of measure algebras.}

Lemma 2. Let $F$ and $G$ be locally compact groups, a a bicontinuous isomorphism of $F$ onto $G$ and $\gamma$ a character on $F$. Let $T$ be the mapping of $M(F)$ into $M(G)$ defined by

$$
T \mu(f)=\mu(\gamma(f \circ \alpha)), \quad \mu \in M(F), f \in C_{0}(G) .
$$

Then $T$ is an isometric *-isomorphism of $M(F)$ onto $M(G)$; and $T$ is a bicontinuous mapping of $M(F)_{\sigma}$ onto $M(G)_{\sigma}$.

Proof. Consider the mapping $f \mapsto \gamma(f \circ \alpha)$ of $C_{0}(G)$ into $C_{0}(F)$. It is easily seen that this mapping is an isometry of $C_{0}(G)$ onto $C_{0}(F)$ and that $T$ is its adjoint. Consequently $T$ is an isometry of $M(F)$ onto $M(G)$ and is a bicontinuous mapping of $M(F)_{\sigma}$ onto $M(G)_{\sigma}$. That $T(\mu * \lambda)=T \mu * T \lambda$ and $(T \mu)^{*}=T \mu *$ hold may be shcwn by calculations.

THEOREM 1. Let $F$ and $G$ be locally compact groups and let $T$ be a norm decreasing isomorphism of $M(F)$ onto $M(G)$. Then there is a bicontinuous isomorphism a of $F$ onto $G$, and a character $\gamma$ on $F$ such that

$$
T \mu(f)=\mu(\gamma(f \circ \alpha)), \quad \mu \in M(F), f \in C_{0}(G) .
$$

Proof. For $x \in F$ we have by Lemma 1, that there is a complex number $\gamma(x)$ with $|\gamma(x)|=1$, and an element $\alpha(x)$ of $G$ such that $T \varepsilon_{x}=\gamma(x) \varepsilon_{\alpha(x)}$. Consider the mappings

$$
\gamma: x \mapsto \gamma(x) \text { and } \alpha: x \mapsto \alpha(x) .
$$

We first show that $\alpha$ is a homomorphism of $F$ into $G$, and $\gamma$ is a homomorphism of $F$ into the complex numbers of absolute value 1 . Clearly $T \varepsilon_{x y}=\gamma(x y) \varepsilon_{\alpha(x y)}$, and

$$
T \varepsilon_{x} * T \varepsilon_{y}=\gamma(x) \gamma(y) \varepsilon_{\alpha(x)} * \varepsilon_{\alpha(y)}=\gamma(x) \gamma(y) \varepsilon_{\alpha(x) \alpha(y)} .
$$

Since $T \varepsilon_{x} * T \varepsilon_{y}=T\left(\varepsilon_{x} * \varepsilon_{y}\right)=T \varepsilon_{x y}$, we have

$$
\gamma(x y) \varepsilon_{\alpha(x y)}=\gamma(x) \gamma(y) \varepsilon_{\alpha(x) \alpha(y)}
$$

and since the Dirac measures are pairwise linearly independent, we have $\gamma(x y)=$ $\gamma(x) \gamma(y)$, and $\alpha(x y)=\alpha(x) \alpha(y)$. Thus $\alpha$ is a homomorphism of $F$ into $G$, and $\gamma$ is a homomorphism of $F$ into the complex numbers. 
We now show that $\gamma$ is continuous. For any nonnegative $f$ in $C_{0}(G)$ we have $\left|T \varepsilon_{x}(f)\right|=\varepsilon_{\alpha(x)}(f)$ consequently $\gamma(x)\left|T \varepsilon_{x}(f)\right|=T \varepsilon_{x}(f)$. Thus to show the continuity of $\gamma$ it suffices to show that the mapping $x \mapsto T \varepsilon_{x}(f)$ is continuous at $e$ in $F$ where $f$ is a nonnegative function in $C_{0}(G)$ such that $T \varepsilon_{e}(f) \neq 0$. By the definition of the weak topology the mapping $T \varepsilon_{x} \mapsto T \varepsilon_{x}(f)$ is continuous from $M(G)_{\sigma}$ into the complex numbers. The mapping $x \mapsto \varepsilon_{x}$ is continuous from $F$ to $F_{s o}^{\varepsilon}$ by Propositions 1 and 2 and the mapping $\varepsilon_{x} \mapsto T \varepsilon_{x}$ is continuous from $F_{s o}^{\varepsilon}$ to $M(G)_{\sigma}$ by Proposition 4. It follows that $\gamma$ is continuous and thus $\gamma$ is a character.

The continuity of $\alpha$ follows by considering the mappings

$$
x \mapsto \varepsilon_{x} \mapsto T \varepsilon_{x}=\gamma(x) \varepsilon_{\alpha(x)} \mapsto \varepsilon_{\alpha(x)} \mapsto \alpha(x) .
$$

The only mapping we have to check is the mapping $\gamma(x) \varepsilon_{\alpha(x)} \mapsto \varepsilon_{\alpha(x)}$, of a subset of $M(G)_{\sigma}$ into $M(G)_{\sigma}$. But since this mapping is multiplication by the continuous character $x \mapsto(\gamma(x))^{-}$, it is continuous. Thus $\alpha$ is continuous since it may be written as a composite of continuous mappings. Moreover since each of the above mappings is one to one we have that $\alpha$ is a continuous one to one homomorphism. Now consider $T^{-1}$. By the open mapping theorem $T^{-1}$ is a bounded isomorphism of $M(G)$ onto $M(F)$. Thus Proposition 4 applies and we have that $\varepsilon_{\alpha(x)} \mapsto T^{-1} \varepsilon_{\alpha(x)}$ $=(\gamma(x))^{-} \varepsilon_{x}$ is continuous from $G_{s o}^{\varepsilon}$ into $M(F)_{\sigma}$. The continuity of $\alpha^{-1}$ restricted to $\alpha(F)$ now follows by considering the mappings

$$
\alpha(x) \mapsto \varepsilon_{\alpha(x)} \mapsto T^{-1} \varepsilon_{\alpha(x)}=(\gamma(x))^{-} \varepsilon_{x} \mapsto \varepsilon_{x} \mapsto x .
$$

Thus $F$ is homeomorphic to $\alpha(F)$ and since a locally compact group is complete $\alpha(F)$ is complete and therefore closed.

Now suppose $\alpha$ is not onto; then there is a $y$ in $G \mid \alpha(F)$ and a compact neighborhood $V$ of $y$ such that $V \cap \alpha(F)=\varnothing$ because $\alpha(F)$ is closed. Since $T^{-1} \varepsilon_{y}$ is in $M(F)$, by Proposition 2 there is a net $\left(\mu_{j}: j \in J\right)$ such that

$$
\begin{aligned}
\mu_{j} & =\sum_{i=1}^{n_{j}} b_{i, j} \varepsilon_{x_{i, j}}, \quad x_{i, j} \in F, b_{i, j} \text { complex, } \\
\left\|\mu_{j}\right\| & \leqq\left\|T^{-1} \varepsilon_{y}\right\|
\end{aligned}
$$

and $\mu_{j} \stackrel{s o}{\rightarrow} T^{-1} \varepsilon_{y}$. Thus by Proposition $4, T \mu_{j} \stackrel{\sigma}{\rightarrow} \varepsilon_{y}$. Note that $T \varepsilon_{x}=\gamma(x) \varepsilon_{\alpha(x)}$, thus

$$
T \mu_{j}=\sum_{i=1}^{n_{j}} b_{i, j} \gamma\left(x_{i, j}\right) \varepsilon_{\alpha\left(x_{i, j}\right)} .
$$

Since $G$ is locally compact there is a function $f$ in $C_{0}(G)$ such that $f(y)=1$ and $f(G \backslash V)=0$ and $0 \leqq f(x) \leqq 1$ for all $x \in G$. Since $T \mu_{j} \stackrel{\sigma}{\rightarrow} \varepsilon_{y}$ we have

$$
\sum_{i=1}^{n_{j}} b_{i, j} \gamma\left(x_{i, j}\right) \varepsilon_{\alpha\left(x_{i, j}\right)}(f) \rightarrow f(y) .
$$

But since $\alpha\left(x_{i, j}\right) \in \alpha(F) \cap(G \backslash V), \quad \varepsilon_{\alpha\left(x_{i, j}\right)}(f)=f\left(\alpha\left(x_{i, j}\right)\right)=0$, so that $f(y)=0$, a contradiction. Thus $\alpha$ is onto. 
All that remains now is to establish the formula $(T \mu)(f)=\mu(\gamma(f \circ \alpha))$.

Let $T_{1}$ be the mapping defined by

$$
\left(T_{1} \mu\right)(f)=\mu(\gamma(f \circ \alpha)), \quad \mu \in M(F), f \in C_{0}(G) .
$$

By Lemma 2 we have that $T_{1}$ is an isomorphism and isometry from $M(F)$ onto $M(G)$. Hence in view of Proposition 4, $T_{1}$ is continuous on norm bounded sets from $M(F)_{s o}$ onto $M(G)_{\sigma}$. Now observe that

$$
T_{1} \varepsilon_{x}(f)=\varepsilon_{x}(\gamma(f \circ \alpha))=\gamma(x) f(\alpha(x))=\gamma(x) \varepsilon_{\alpha(x)}(f)=T \varepsilon_{x}(f)
$$

Thus $T$ and $T_{1}$ coincide on $F^{\varepsilon}$, and by Proposition 2 each $\mu \in M(F)$ is a so-adherence point of a norm bounded set of linear combinations of Dirac measures so we have $T=T_{1}$. This completes the proof.

COROLlaRY 1. Every norm decreasing isomorphism of $M(F)$ onto $M(G)$ is an isometric *-isomorphism.

Proof. This follows from Lemma 2 and the above theorem.

COROllaRY 2. Let $T$ be a norm decreasing isomorphism of $M(F)$ onto $M(G)$; then $T$ is a bicontinuous mapping of $M(F)_{\sigma}$ onto $M(G)_{\sigma}$.

Proof. This follows from Lemma 2 and the above theorem.

COROllaRY 3. Each norm decreasing isomorphism of $M(F)$ onto $M(G)$ maps $L^{1}(F)$ onto $L^{1}(G)$.

Proof. This follows from the formula.

COROLlaRY 4. $L^{1}(G)$ is invariant under norm decreasing automorphisms of $M(G)$.

The following example shows that a $*$-isomorphism of $M(F)$ onto $M(G)$ need not be norm decreasing. Let $F$ and $G$ be finite abelian groups of order $n$, and suppose that $F$ and $G$ are not isomorphic. Let $\gamma_{1}, \gamma_{2}, \ldots, \gamma_{n}$ be the $n$ characters of $F$ and define functions $f_{i}, i=1,2, \ldots, n$ on $F$ by

$$
f_{i}(x)=(1 / n) \gamma_{i}(x), \quad x \in F, i=1,2, \ldots, n .
$$

Using the orthogonality relations for characters on abelian groups it follows that $f_{1}, f_{2}, \ldots, f_{n}$ are orthogonal idempotents which span $M(F)$. Clearly $f_{i}^{*}=f_{i}$. Let $g_{1}, g_{2}, \ldots, g_{n}$ be a basis for $M(G)$ defined in the analogous manner. Define $T$ by $T f_{i}=g_{i}, i=1,2, \ldots, n$ and extend $T$ to a linear mapping of $M(F)$ into $M(G)$. It is easily seen that $T$ is a $*$-isomorphism of $M(F)$ onto $M(G) . T$ cannot be norm decreasing if $F$ and $G$ are not isomorphic since this would violate Theorem 1.

As a consequence of Theorem 1 we shall derive a theorem due to Wendel [8] on isomorphisms of $L^{1}(F)$ onto $L^{1}(G)$. First we shall need the following lemma.

Lemma 3. Let $T$ be a bounded isomorphism of $L^{1}(F)$ onto $L^{1}(G)$. Then there is a unique bounded isomorphism $T^{\#}$ of $M(F)$ onto $M(G)$ which extends $T$. Moreover, $\|T \#\|=\|T\|$. 
Proof. Clearly $T$ is continuous as a mapping of $L^{1}(F)_{s o}$ onto $L^{1}(G)_{s o}$. Since $M(G)_{s o}$ is quasi-complete (see the remark preceding Proposition 2) and since each $\mu$ in $M(F)$ is a so-adherence point of a bounded set in $L^{1}(F)$ (Proposition 2), $T$ has a unique extension $T^{\#}$ to a continuous linear mapping of $M(F)_{s o}$ into $M(G)_{s o}$ [1, Chapitre III, §2, No. 5].

To show that $T^{\#}(\mu * \lambda)=T^{\#} \mu * T^{\#} \lambda, \mu, \lambda$ in $M(F)$, let $\left(\mu_{j}: j \in J\right)$ and $\left(\lambda_{k}: k \in K\right)$ be nets in $L^{1}(F)$ such that $\mu_{j} \stackrel{\text { so }}{\rightarrow} \mu$ and $\lambda_{j} \stackrel{s o}{\rightarrow} \lambda$. Then since multiplication is separately continuous in $M(F)_{s o}$ (Proposition 2) we have

$$
\mu * \lambda=\lim _{j}\left(\lim _{k} \mu_{j} * \lambda_{k}\right) .
$$

Therefore the so-continuity of $T^{\#}$ implies that

$$
T^{\#}(\mu * \lambda)=\lim _{j}\left(\lim _{k} T\left(\mu_{j} * \lambda_{k}\right)\right) .
$$

Since $T^{\#}=T$ on $L^{1}(F)$ we have $T^{\#}\left(\mu_{j} * \lambda_{k}\right)=T \mu_{j} * T \lambda_{k}$. Now using the fact that multiplication is separately continuous in $M(G)_{s o}$ we have

$$
\lim _{j}\left(\lim _{k} T \mu_{j} * T \lambda_{k}\right)=T^{\#} \mu * T^{\#} \lambda .
$$

Combining the above we have $T^{\#}(\mu * \lambda)=T^{\#} \mu * T^{\#} \lambda$.

To show that $T^{\#}$ is one-one, let $\lambda, \mu \in M(F)$ and suppose $T^{\#} \mu=T^{\#} \lambda$. If $\lambda \neq \mu$ then there is a $\nu$ in $L^{1}(F)$ such that $\lambda * \nu \neq \mu * \nu$. Then $T(\lambda * \nu)=T^{\#} \lambda * T \nu=T^{\#} \mu * T \nu$ $=T(\mu * \nu)$ which contradicts the assertion that $T$ is an isomorphism because $\lambda * \nu$ and $\mu * \nu$ are in $L^{1}(F)$. Therefore $T^{\#}$ is one-one.

We now show that $T^{\#}$ is onto. Let $\mu^{\prime} \in M(G)$, by Proposition 2 there is a net $\left(\mu_{j}^{\prime}: j \in J\right)$ in $L^{1}(G)$ such that $\mu_{j}^{\prime} \stackrel{s o}{\rightarrow} \mu^{\prime}$ and $\left\|\mu_{j}^{\prime}\right\| \leqq\left\|\mu^{\prime}\right\|$. By the open mapping theorem $T^{-1}$ is bounded, so that $\left(T^{-1} \mu_{j}^{\prime}: j \in J\right)$ is a bounded Cauchy net in $L^{1}(F)_{s o}$. Since $M(F)_{s o}$ is quasi-complete there is a $\mu$ in $M(F)$ such that $T^{-1} \mu_{j}^{\prime} \stackrel{s o}{\rightarrow} \mu$. Then $T\left(T^{-1} \mu_{j}^{\prime}\right) \stackrel{\text { so }}{\rightarrow} T \mu$ so that $T^{\#} \mu=\mu^{\prime}$ and $T^{\#}$ is onto.

We next show $\left\|T^{\#}\right\|=\|T\|$. Clearly $\|T\| \leqq\|T \#\|$. To show the reverse inequality let $\mu \in M(F)$ be given. By Proposition 2 there is a net $\left(\mu_{j}: j \in J\right)$ in $L^{1}(F)$ such that $\mu_{j} \stackrel{\text { so }}{\rightarrow} \mu$ and $\left\|\mu_{j}\right\| \leqq\|\mu\|$ so that $T \mu_{j} \stackrel{\text { so }}{\longrightarrow} T^{\#} u$. Since the mapping $\lambda \mapsto\|\lambda\|$ is lower semicontinuous in the so-topology (Corollary 1 to Proposition 2), we have $\left\|T^{\#} \mu\right\|$ $\leqq \lim \inf \left\|T_{j}\right\| \leqq \lim \inf \|T\|\left\|\mu_{j}\right\| \leqq\|T\|\|\mu\|$. Therefore $\left\|T^{\# \|} \leqq\right\| T \|$ and hence $\left\|T^{\#}\right\|=\|T\|$.

The uniqueness of $T^{\#}$ is an easy consequence of Propositions 2 and 4.

THEOREM 2 (WENDEL). Let $T$ be a norm decreasing isomorphism of $L^{1}(F)$ onto $L^{1}(G)$. Then there is a bicontinuous isomorphism $\propto$ of $F$ onto $T$, a character $\gamma$ on $G$ such that

$$
T \lambda(f)=\lambda(\gamma(f \circ \alpha)) \quad \lambda \in L^{1}(F), f \in C_{0}(G) .
$$

Proof. This follows easily from Theorem 1 in virtue of Lemma 3.

COROLlaRY. Each norm decreasing isomorphism of $L^{1}(F)$ onto $L^{1}(G)$ is an isometric $*$-isomorphism. 
4. Norm decreasing homomorphisms of measure algebras. In this section we give the structure of all norm decreasing homomorphisms $T$ of $M(F)$ onto $M(G)$ such that for $\mu \in M(F), T\left(\mu * L^{1}(F)\right)=\{0\}$ implies $T \mu=0$.

Lemma 4. Let $F$ and $G$ be locally compact groups, $\alpha$ a continuous and open homomorphism of $F$ onto $G$, and $\gamma$ a character on $F$. Let $T$ be the mapping of $M(F)$ into $M(G)$ defined by

$$
T \mu(f)=\mu(\gamma(f \circ \alpha)) \quad \mu \in M(F), f \in C_{0}(G) .
$$

Then $T$ is a norm decreasing *-homomorphism of $M(F)$ onto $M(G) ; T$ is continuous as a mapping of $M(F)_{\text {so }}$ onto $M(G)_{s o}$ and $T\left(L^{1}(F)\right)=L^{1}(G)$.

Proof. Let $e$ be the unit of $G$ and let $F_{0}=\{x: \alpha(x)=e\}$. Then $F_{0}$ is a closed normal subgroup of $F$. Let $\pi: F \rightarrow F / F_{0}$ be the canonical mapping then there is a bicontinuous isomorphism $\beta: F / F_{0} \rightarrow G$ such that $\alpha=\beta \circ \pi$. Consider the following mappings:

(1) $\gamma: M(F) \rightarrow M(F)$ defined by $\gamma \mu(f)=\mu(\gamma f) \mu \in M(F), f \in C_{0}(F)$,

(2) $\pi^{* *}: M(F) \rightarrow M\left(F / F_{0}\right)$ defined by $\pi^{* *} \mu(f)=\mu(f \circ \pi) \mu \in M(F), f \in C_{0}\left(F / F_{0}\right)$,

(3) $\beta^{* *}: M\left(F / F_{0}\right) \rightarrow M(G)$ defined by $\beta^{* *} \mu(f)=\mu(f \circ \beta) \mu \in M\left(F / F_{0}\right), f \in C_{0}(G)$.

By Lemma 2, (1) and (3) are isometric isomorphisms. By Lemma 5.1.8 of Greenleaf [4], $\pi^{* *}$ is a norm decreasing homomorphism of $M\left(F / F_{0}\right)$ onto $M(G)$. Observe that $T=\beta^{* *} \pi^{* *} \gamma$ so that $T$ is a norm decreasing homomorphism of $M(F)$ onto $M(G)$. That $T$ is a $*$-homomorphism follows by a calculation. To complete the proof it suffices to show that $T\left(L^{1}(F)\right)=L^{1}(G)$. In the proof of Theorem 5.2.1 of [4] Greenleaf shows that $\pi^{* *}\left(L^{1}(F)\right)=L^{1}\left(F / F_{0}\right)$ consequently by Corollary 3 to Theorem 1 , $T\left(L^{1}(F)\right)=L^{1}(G)$.

The next lemma is an observation of Greenleaf's [4] and [5].

LEMMA 5. Let $F$ and $G$ be locally compact groups; $\alpha$ a continuous homomorphism of $F$ into $G$ and $\gamma$ a character of $F$. Then the mapping $T$ defined by $T \mu(f)=\mu(\gamma(f \circ \alpha))$ is a norm decreasing homomorphism of $M(F)$ into $M(G)$ which is continuous on norm bounded sets as a mapping of $M(F)_{\text {so }}$ into $M(G)_{\sigma}$. If $\alpha$ is a monomorphism then so is $T$.

Proof. It is straightforward to verify that $T$ is a norm decreasing homomorphism of $M(F)$ into $M(G)$. The stated continuity property of $T$ follows from Lemma 1.1.1 of Greenleaf [4] and the fact that for $f \in C_{0}(G), f \circ \alpha$ is left (and right) uniformly continuous on $F$. If $\alpha$ is a monomorphism then to show that $T$ is a monomorphism it suffices to show that the mapping $\alpha^{* *}$ defined by $\alpha^{* *} \mu(f)=\mu(f \circ \alpha), \mu \in M(F)$, $f \in C_{0}(G)$ is a monomorphism. To show this let $K \subseteq F$ be any compact subset, then $\mu(K)=\mu\left(\alpha^{-1} \alpha(K)\right)=\alpha^{* *} \mu(\alpha(K))$ since $\alpha$ is a monomorphism. It follows that $\alpha^{* *} \mu=0$ implies $\mu=0[6$, p. 175]. This completes the proof. 
THEOREM 3. Let $T$ be a norm decreasing homomorphism of $M(F)$ onto $M(G)$. If $T\left(\mu * L^{1}(F)\right)=\{0\}$ implies $T \mu=0$, then there is a character $\gamma$ on $F$ and a continuous and open homomorphism $\alpha$ of $F$ onto $G$ such that

$$
T \mu(f)=\mu(\gamma(f \circ \alpha)) \text { for } \mu \in M(F), f \in C_{0}(G) .
$$

Proof. For $x \in F$ we have by Lemma 1, a complex number $\gamma(x),|\gamma(x)|=1$ and an $\alpha(x) \in G$ such that $T \varepsilon_{x}=\gamma(x) \varepsilon_{\alpha(x)}$. It follows as in the proof of Theorem 1 that $\alpha$ is a continuous homomorphism of $F$ into $G ; \gamma$ is a character of $F$ and that $T \mu(f)$ $=\mu(\gamma(f \circ \alpha)$ ) (in virtue of Lemma 5). To complete the proof it remains to show that $\alpha$ is open and onto. Let $e$ be the unit of $G$ and let $F_{0}=\{x: \alpha(x)=e\}$. Let $\pi: F \rightarrow F / F_{0}$ be the canonical homomorphism and define $T_{1}: M(F) \rightarrow M\left(F / F_{0}\right)$ by $T_{1} \mu(f)$ $=\mu(\gamma(f \circ \pi))$. By Lemma $4, T_{1}$ is a norm decreasing homomorphism which is onto. Let $\mu \in \operatorname{ker} T_{1}$, then $\mu(\gamma(f \circ \pi))=0$ for all $f$ in $C_{0}\left(F / F_{0}\right)$ and it follows from Lemma 5.2.2 of Greenleaf [4], that $\mu(\gamma(f \circ \pi))=0$ for all $f \in C\left(F / F_{0}\right)$. Let $\beta: F / F_{0} \rightarrow G$ be such that $\alpha=\beta \circ \pi$. For $f \in C_{0}(G), f \circ \beta \in C\left(F / F_{0}\right)$ so that $\mu(\gamma(f \circ \beta \circ \pi))=0$. Thus we have that $\operatorname{ker} T_{1} \subseteq \operatorname{ker} T$ so we may define a homomorphism $T_{2}: M\left(F / F_{0}\right)$ $\rightarrow M(G)$ by $T_{2}\left(T_{1} \mu\right)=T \mu$. To show that $T_{2}$ is an isomorphism we need only show that $T_{2}$ is a monomorphism since $T_{2}$ is clearly onto. Define $T^{\prime}: M\left(F / F_{0}\right) \rightarrow M(G)$ by $T^{\prime} \mu(f)=\mu(f \circ \beta)$, then $T^{\prime} T_{1} \mu(f)=T_{1} \mu(f \circ \beta)=\mu(\gamma(f \circ \beta \circ \pi))=T \mu(f)=T_{2} T_{1} \mu(f)$ for all $\mu$ in $M(F)$. Since $T$ and $T_{1}$ are onto, $T_{2}=T^{\prime}$. Now since $\beta$ is a continuous monomorphism, $T_{2}$ is a norm decreasing monomorphism (Lemma 5).

If we now apply Theorem 1 to $T_{2}$ we have that there is a character $\gamma^{\prime}$ on $F / F_{0}$ and a bicontinuous isomorphism $\alpha^{\prime}$ of $F / F_{0}$ onto $G$ such that

$$
T_{2}\left(T_{1} \mu\right)(f)=T_{1} \mu\left(\gamma^{\prime}\left(f \circ \alpha^{\prime}\right)\right), \quad \mu \in M(F) \text { and } f \in C_{0}(G) .
$$

Thus using the definition of $T_{1}$ we have

$$
\mu\left(\gamma\left(\gamma^{\prime} \circ \pi\right)\left(f \circ \alpha^{\prime} \circ \pi\right)\right)=\mu(\gamma(f \circ \alpha)), \quad \mu \in M(F) \text { and } f \in C_{0}(G) .
$$

It follows that $\alpha^{\prime} \circ \pi=\alpha$ and consequently $\alpha$ is open and onto.

COROLlaRY 1. Let $T$ be a norm decreasing homomorphism of $M(F)$ onto $M(G)$. If $T\left(\mu * L^{1}(F)\right)=\{0\}$ implies $T \mu=0$, then $T\left(L^{1}(F)\right)=L^{1}(G)$ and consequently $T$ is continuous as a mapping of $M(F)_{\text {so }}$ onto $M(G)_{s o}$.

Proof. This follows from the theorem and Lemma 4.

COROLlaRY 2. Each norm decreasing homomorphism of $M(F)$ onto $M(G)$ satisfying the hypothesis of Theorem 3 is a *-homomorphism.

REMARK. There are homomorphisms $T$ which are onto but which do not satisfy the hypothesis of the theorem as the following example shows.

Let $F$ be a nondiscrete locally compact group and let $G=\{e\}$ be the group consisting of only one element. Define $T: M(F) \rightarrow M(G)$ by $T \mu=\sum_{x \in F} \mu(\{x\}) \varepsilon_{e}$. Then $T$ 
is a norm decreasing homomorphism which is onto and $T^{\prime}$ annihilates $L^{1}(F)$. Corollary 1 to the theorem shows that the conclusion of Theorem 2 cannot hold for $T$.

\section{Bipositive homomorphisms of measure algebras.}

Definition. A homomorphism $T$ of $M(F)$ onto $M(G)$ is said to be bipositive if $T$ maps the set of positive measures onto the set of positive measures.

THEOREM 4. Let $T$ be a bipositive homomorphism of $M(F)$ onto $M(G)$. If $T\left(\mu * L^{1}(F)\right)=\{0\}$ implies $T \mu=0$ then there is a continuous and open homomorphism $\alpha$ of $F$ onto $G$ such that

$$
T \mu(f)=\mu(f \circ \alpha), \quad \mu \in M(F), f \in C_{0}(G) .
$$

If $T$ is an isomorphism then so is $\alpha$.

Proof. Note that a positive measure $\mu$ on $G$ is a real multiple of a Dirac measure if and only if each $\lambda \in M(G)$ for which $\mu-\lambda$ is a positive measure is a real multiple of $\mu$. Since this property is clearly preserved under bipositive maps we have that for each $x \in F$, there is a real number $\gamma(x)$ and an $\alpha(x) \in G$ such that $T \varepsilon_{x}=\gamma(x) \varepsilon_{\alpha(x)}$. It is readily verified that the mapping $x \mapsto \gamma(x)$ is a homomorphism of $G$ into the positive reals. Since $T$ is positive, $T$ is bounded, thus there is a constant $M$ such that $\|T \mu\|$ $\leqq M\|\mu\|$, for all $\mu \in M(F)$. It follows that $\{\gamma(x): x \in G\}$ is a bounded subgroup of the positive reals. Thus we must have $\gamma(x)=1$ for all $x \in F$. Applying Proposition 4 we see that $T \mu(f)=\mu(f \circ \alpha)$ for $\mu \in M(F), f \in C_{0}(G)$. By Lemma $5, T$ is a norm decreasing homomorphism and Theorems 1 and 3 now apply.

COROllaRY. Let $T$ be a bipositive homomorphism of $M(F)$ onto $M(G)$. If $T\left(\mu * L^{1}(F)\right)=\{0\}$ implies $T \mu=0$ then $T$ is a norm decreasing *-homomorphism.

REMARK. The example given after Corollary 2 to Theorem 3 shows that there are bipositive homomorphisms which do not satisfy the hypothesis of the theorem.

The author has recently learned that G. Gaudry has proven Theorem 4 for the case when $T$ is a bipositive isomorphism (to appear, Canad. J. Math.).

\section{REFERENCES}

1. N. Bourbaki, Éléments de mathématique. Livre V. Espaces vectoriels topologiques, Chapitres I-V, Hermann, Paris, 1953-1955.

2. —_ Éléments de mathématique. Livre VI. Intégration, Chapitres I-IV, Hermann, Paris, 1965.

3. N. Dunford and J. Schwartz, Linear operators. Part I: General theory, Interscience, New York, 1958.

4. F. Greenleaf, Norm decreasing homomorphisms of group algebras, Pacific J. Math. 15 (1965), 1187-1220.

5. — "Closed subalgebras of group algebras which are group algebras" in Function algebras, Proc. Internat. Sympos. on Function Algebras, Tulane Univ., 1965, Scott-Foresman, Chicago, Ill., 1966, pp. 276-281. 
6. E. Hewitt and K. Ross, Abstract harmonic analysis. I, Springer-Verlag, Berlin, 1963.

7. B. E. Johnson, Isometric isomorphisms of measure algebras, Proc. Amer. Math. Soc. 15 (1964), 186-188.

8. J. G. Wendel, Left centralizers and isomorphisms of group algebras, Pacific J. Math. 2 (1952), 251-261.

MCMASTER UNIVERSITY,

Hamilton, Canada

MCGILL UNIVERSITY,

Montreal, CANADA 